\title{
Urinary dysfunction and orthostatic hypotension in multiple system atrophy: which is the more common and earlier manifestation?
}

Ryuji Sakakibara, Takamichi Hattori, Tomoyuki Uchiyama, Kohei Kita, Masato Asahina, Atsuya Suzuki, Tomonori Yamanishi

\begin{abstract}
Objectives-Urinary dysfunction and orthostatic hypotension are the prominent autonomic features in multiple system atrophy (MSA). A detailed questionnaire was given and autonomic function tests were performed in 121 patients with MSA concerning both urinary and cardiovascular systems.
\end{abstract}

Methods-Replies to the questionnaire on autonomic symptoms were obtained from 121 patients including three clinical variants; olivopontocerebellar atrophy (OPCA) type in 48, striatonigral degeneration (SND) type in 17, and Shy-Drager type in 56. Urodynamic studies comprised measurement of postmicturition residuals, EMG cystometry, and bethanechol injection. Cardiovascular tests included head up tilt test, measurement of supine plasma noradrenaline (norepinephrine,NA), measurement of $R-R$ variability (CV R-R), and intravenous infusions of NA and isoproterenol.

Results-Urinary symptoms (96\%) were found to be more common than orthostatic symptoms $(43 \%)(p<0.01)$ in patients with MSA, particularly with OPCA $(p<0.01)$ and SND $(p<0.01)$ types. In 53 patients with both urinary and orthostatic symptoms, patients who had urinary symptoms first $(48 \%)$ were more common than those who had orthostatic symptoms first $(29 \%)$, and there were patients who developed both symptoms simultaneously (23\%). Post-micturition residuals were noted in $74 \%$ of the patients. EMG cystometry showed detrusor hyperreflexia in $56 \%$, low compliance in $31 \%$, atonic curve in $5 \%$, detrusor-sphincter dyssynergia in $45 \%$, and neurogenic sphincter EMG in $74 \%$. The cystometric curve tended to change from hyperreflexia to low compliance, then atonic curve in repeated tests. Bethanechol injection showed denervation supersensitivity of the bladder in $19 \%$. Cardiovascular tests showed orthostatic hypotension below -30 $\mathrm{mm} \mathrm{Hg}$ in $41 \%$, low CV R-R below 1.5 in $57 \%$, supine plasma NA below $100 \mathrm{pg} / \mathrm{ml}$ in $28 \%$, and denervation supersensitivity of the vessels ( $\alpha$ in $73 \% ; \beta 2$ in $60 \%$ ) and of the heart ( $\beta 1$ in $62 \%)$.

Conclusion-It is likely that urinary dysfunction is more common and often an earlier manifestation than orthostatic hy- potension in patients with MSA, although subclinical cardiovascular abnormalities appear in the early stage of the disease. The responsible sites seem to be central and peripheral for both dysfunctions. (f Neurol Neurosurg Psychiatry 2000;68:65-69)

Keywords: autonomic dysfunction; multiple system atrophy; orthostatic hypotension; urinary incontinence; urodynamic study

Multiple system atrophy (MSA)c is known to have urinary disturbance. ${ }^{12}$ Some male patients with MSA have undergone urological surgery for prostatic hypertrophy before the correct diagnosis had been made. The results of the surgery are often transient or unfavourable because of the progressive nature of this disease. Previously we investigated urinary function in 86 patients with MSA considering three clinical variants; striatonigral degeneration (SND), sporadic olivopontocerebellar atrophy (OPCA), and the Shy-Drager syndrome. ${ }^{3}$ The study showed that $4 \%$ of the patients with OPCA type, $16 \%$ with SND type, and $50 \%$ with Shy-Drager type had urinary symptoms at the onset of disease, and in particular $17 \%$ of the patients with Shy-Drager type had urinary symptoms as the sole initial symptom. Orthostatic hypotension was pointed out first in patients with Shy-Drager type, ${ }^{4}$ which turned out to be a marker of autonomic involvement in MSA. ${ }^{56}$ Both of the original two patients had urinary frequency, incontinence, and urinary retention as well. ${ }^{4}$ The other two variants rarely develop orthostatic hypotension in their early stage; however, both the patients with OPCA type had voiding difficulty and urinary incontinence, ${ }^{7}$ and three of four with SND type showed voiding difficulty, retention, and urinary incontinence. ${ }^{8}$ In this paper we present data from a detailed questionnaire and autonomic function tests in 121 patients with MSA concerning both urinary and cardiovascular systems.

\section{Patients and methods}

PATIENTS AND AUTONOMIC QUESTIONNAIRE The autonomic questionnaire comprised urinary symptoms including nocturnal and diurnal urinary frequency, sensation of urgency, urinary incontinence, voiding difficulty and retention, and orthostatic symptoms including postural faintness, blurred vision, and syncope. We gave the questionnaire to 121 patients with a clinical diagnosis of MSA, ${ }^{910}$ including 74 


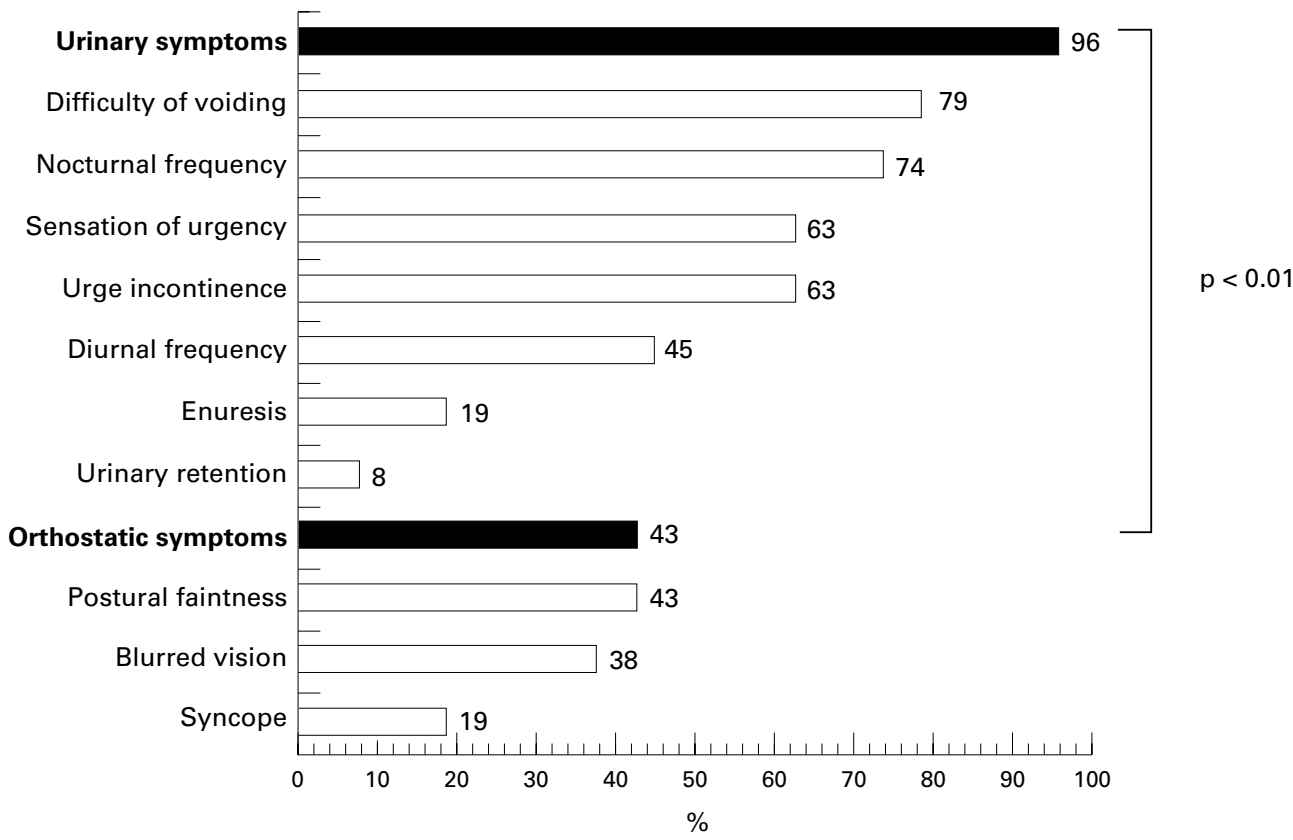

Figure 1 Urinary and orthostatic symptoms in patients with MSA.

men and 47 women, mean age 58 (SD 8.0) years). We also divided the patients into three variants; OPCA type in 48 , SND type in 17 , and Shy-Drager type in 56. In the OPCA type, cerebellar ataxia is the initial and main clinical feature that may be gradually overlapped by parkinsonism during the course of disease. In the SND type, parkinsonism is the initial and main clinical feature throughout the course of the disease. In the Shy-Drager type, autonomic symptoms are the initial and main clinical feature and mild parkinsonism, cerebellar ataxia, and/or pyramidal involvement appear during the course of the disease in all patients, which enables the exclusion of pure autonomic failure. ${ }^{56}$ The duration of the disease was $1-2$ years in 44, 3-4 years in 39, and over 5 years in 38. None had abnormalities on ECG or chest radiography, and in blood chemistry (including blood sugar), urinalysis, or abdominal ultrasonography (including kidney and prostate). The normal ranges of the cardiovascular function tests were obtained from 11 neurologically

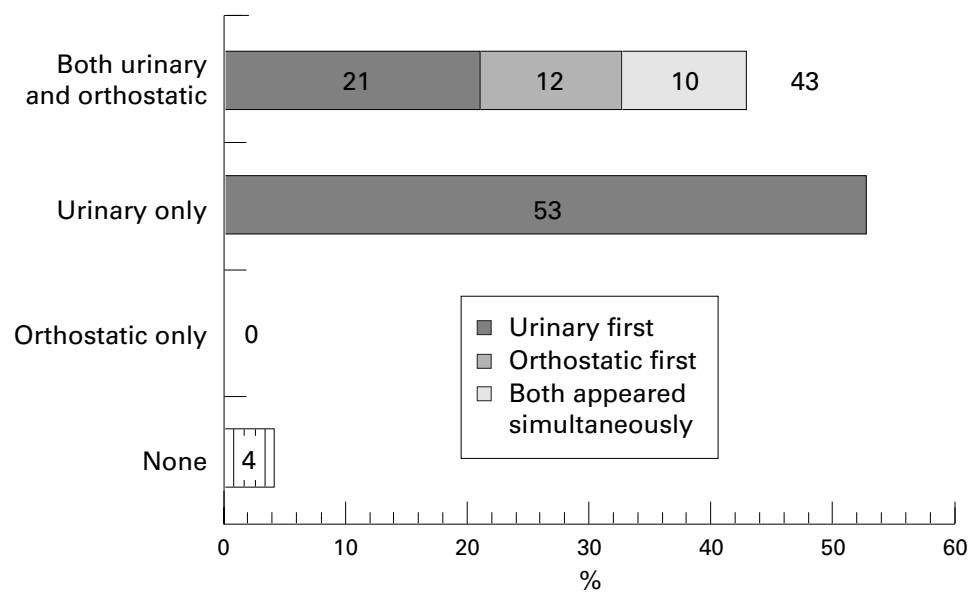

Figure 2 Timing of urinary and orthostatic symptoms in patients with MSA. normal control subjects aged 55 (SD 9.9) years ${ }^{11}$ and the previous reports..$^{12}$

\section{URODYNAMIC STUDIES AND CARDIOVASCULAR} TESTS

After voluntary voiding we measured postmicturition residuals, and their normal volume is under $30 \mathrm{ml}$. The EMG cystometry, medium fill $(50 \mathrm{ml} / \mathrm{min})$ water cystometry with simultaneous sphincter EMG was done with a electromyographic computer (Nihon Kohden; Neuropack $\Sigma$ ) and a urodynamic computer (Lifetech; Janus). The filling phase abnormalities included detrusor hyperreflexia which is a phasic contraction with a detrusor pressure rise ( $\Delta$ Pdet) over $10 \mathrm{~cm} \mathrm{H}_{2} \mathrm{O}$, and low compliance bladder which is a tonic contraction of the detrusor with bladder capacity / $\Delta$ Pdet under $20 \mathrm{ml} / \mathrm{cm} \mathrm{H}_{2} \mathrm{O}$. An atonic curve is defined when the patient has a bladder capacity over $600 \mathrm{ml}$ and is unable to contract the detrusor on voiding. Detrusor-sphincter dyssynergia is defined when the patient is unable to relax the sphincter on voiding. The methods and definitions used for the urodynamic studies conformed to the standards proposed by the International Continence Society. ${ }^{14}$ Seventeen of the patients had repeated urodynamic studies. Analysis of sphincter EMG was performed in 70 patients. Neurogenic sphincter EMG is defined as long duration over $10 \mathrm{~ms}$, polyphase more than five phases, and high amplitude over $600 \mu \mathrm{V}$. A bethanechol test was performed in 53 patients. After infusion of $100 \mathrm{ml}$ water, 2.5 mg bethanechol chloride was injected subcutaneously and detrusor pressure was measured for 30 minutes. $\Delta$ Pdet over $15 \mathrm{~mm} \mathrm{H}_{2} \mathrm{O}$ during the test is considered to be high. ${ }^{15}$

The head up tilt test (80 degrees, 10 minutes) was carried out while monitoring blood pressure (BP) and heart rate (HR) with an automatic sphygmomanometer (Nihon 
Orthostatic symptom

Urinary symptom

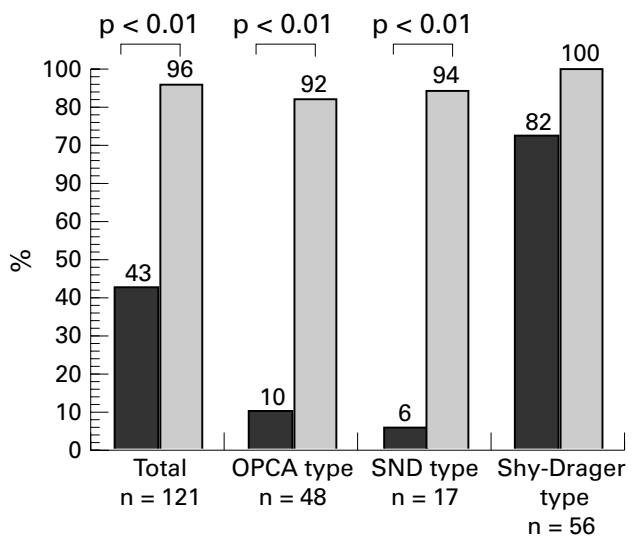

Figure 3 Urinary and orthostatic symptoms in three variants of MSA.

Kohlin; BP-203Y). Systolic blood pressure fall ( $\triangle$ BPsys) was -0.2 (SD 10.7) $\mathrm{mm} \mathrm{Hg}$ in the control group, and below $-30 \mathrm{~mm} \mathrm{Hg}$ is regarded as low considering its variation with age. Supine plasma noradrenaline (norepinephrine) (NA) was measured in 88 patients. It was 188.8 (SD 80.4) $\mathrm{pg} / \mathrm{ml}$ in the control group, and below $100 \mathrm{pg} / \mathrm{ml}$ is regarded as low. $\mathrm{R}-\mathrm{R}$ variability (CV R-R) was measured in 98 patients by an arrhythmia computer (ME Commercial; Automatic R-100). CV R-R was 3.37 (SD 0.55) in the control group, and below 1.5 is regarded as low considering its variation with age. Infusion of NA was performed in 89 patients at a rate of $0.05 \mathrm{mg} / \mathrm{kg} / \mathrm{min}$ for $3 \mathrm{~min}$ -

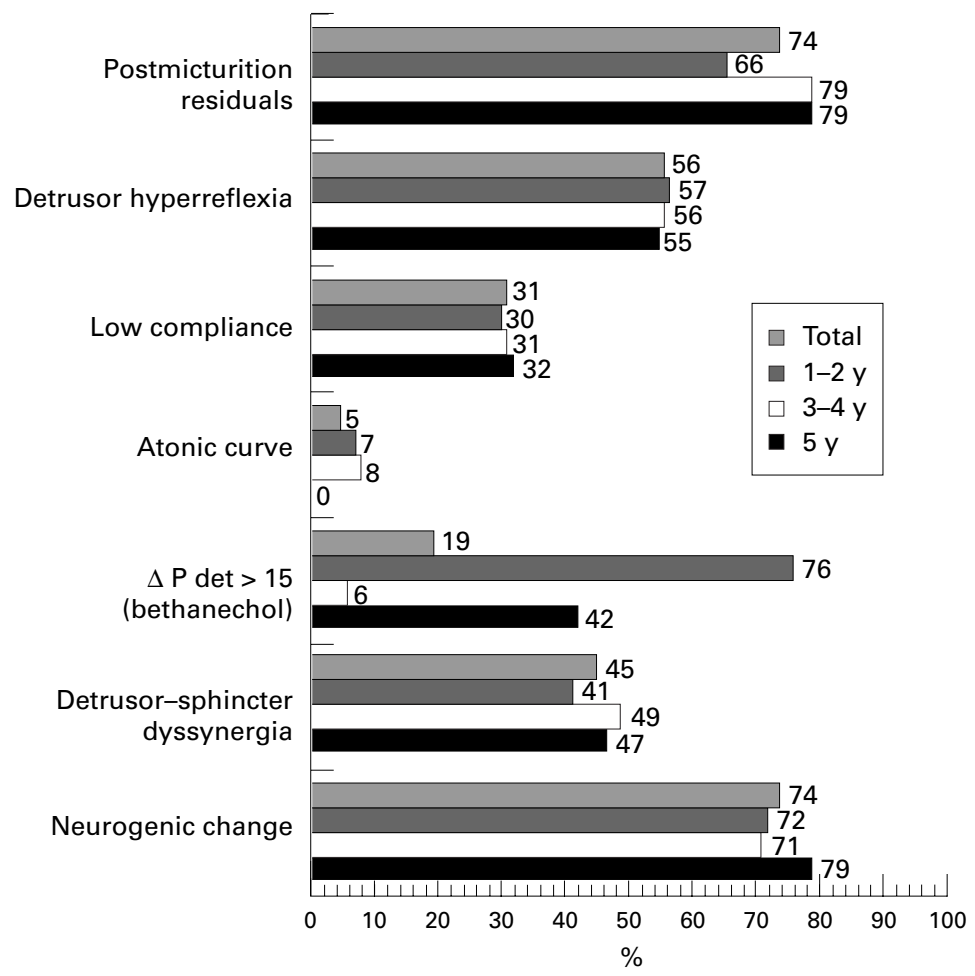

Figure 4 Results of urodynamic studies in patients with MSA. utes and $0.1 \mathrm{mg} / \mathrm{kg} / \mathrm{min}$. for the following 3 minutes to avoid extreme hypertension. $\triangle B$ Psys during the infusion was 10.4 (SD 6.2) $\mathrm{mm} \mathrm{Hg}$ in the control group, and over $30 \mathrm{~mm}$ $\mathrm{Hg}$ is considered to be high. Isoproterenol infusion was performed in 85 patients at a rate of $0.02 \mathrm{mg} / \mathrm{kg} / \mathrm{min}$ for 5 minutes. Change in heart rate $(\Delta \mathrm{HR})$ during the infusion was 38.8 (SD 11)/min in the control group, and over $50 / \mathrm{min}$ is considered to be high. $\Delta \mathrm{BP}$ sys during the infusion was -6.7 (SD 9.8) $\mathrm{mm} \mathrm{Hg}$ in the control group, and below $-20 \mathrm{~mm} \mathrm{Hg}$ is considered to be low.

\section{Results}

The autonomic questionnaire showed that $96 \%$ of the patients had urinary symptoms (figures 1 and 2). The most frequent symptom was difficulty of voiding in $79 \%$, followed by nocturnal urinary frequency in $74 \%$, sensation of urgency in $63 \%$, and urge incontinence in $63 \%$, and urinary retention was noted in $8 \%$. Orthostatic symptoms were found in $43 \%$ of the patients, including postural faintness in $43 \%$, blurred vision in $38 \%$, and syncope in $19 \%$. Urinary symptoms were more common than orthostatic symptoms $(p<0.01)$. In $43 \%$ of patients with both urinary and orthostatic symptoms, patients who had urinary symptoms first $(21 \%)$ were more common than those who had orthostatic symptoms first $(12 \%)$, although there was no statistical significance, and there were patients who developed both symptoms simultaneously (10\%). Fifty three per cent of the patients had only urinary symptoms but none had only orthostatic symptoms. In three variants of MSA, the difference between urinary and orthostatic symptoms was prominent in patients with OPCA type (urinary symptoms in $92 \%$ and orthostatic symptoms in $10 \% ; \mathrm{p}<0.01)$ and with SND type $(94 \%$ and $6 \%$, respectively; $\mathrm{p}<0.01$ ) compared with those with Shy-Drager type $(100 \%$ and $82 \%$, respectively, fig 3).

Results of urodynamic studies showed postmicturition residuals in $74 \%$ of the patients, and those over $100 \mathrm{ml}$ were noted in $52 \%$ (fig 4). EMG cystometry showed detrusor hyperreflexia in $56 \%$, low compliance bladder in $31 \%$, atonic curve in $5 \%$, detrusor-sphincter dyssynergia in $45 \%$, and neurogenic change in $74 \%$ of the patients. Bethanechol injection showed $\Delta$ Pdet over $15 \mathrm{~mm} \mathrm{H}_{2} \mathrm{O}$ in $19 \%$ of the patients. Repeated urodynamic studies of 17 patients showed cystometric findings changing from hyperreflexia to low compliance bladder in eight patients (after 2-3 years), and then one of them developed an atonic curve. The cystometric curve also changed from normal to low compliance bladder in three (after 2 years). Two patients initially with a negative bethanechol test developed denervation supersensitivity (after 2-3 years). Sphincter EMG changed from normal to detrusor-sphincter dyssynergia in five patients (after 2-4 years), and from normal to neurogenic in two (after 2 years). Results of cardiovascular tests showed orthostatic hypotension below $-30 \mathrm{~mm} \mathrm{Hg}$ in $41 \%$, low CV R-R below 1.5 in $57 \%$, and supine plasma NA below $100 \mathrm{pg} / \mathrm{ml}$ in $28 \%$ of the 


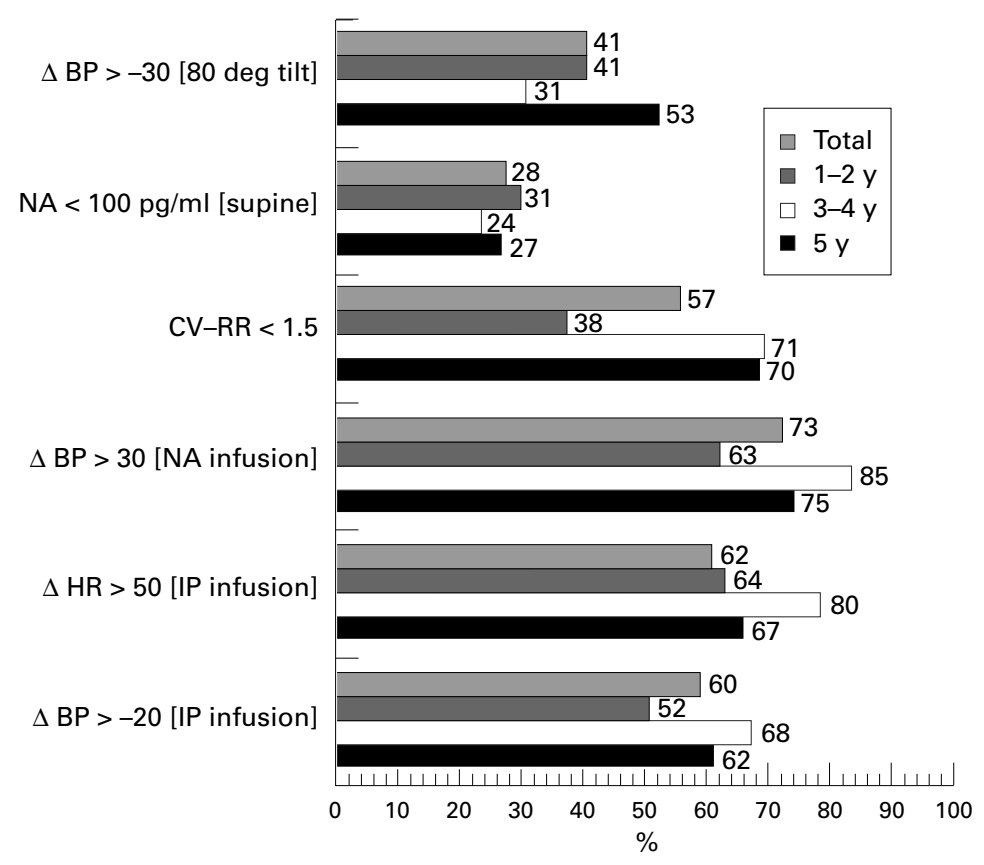

Figure 5 Results of cardiovascular tests in patients with MSA.

patients (fig 5). Infusion of NA showed $\Delta$ BPsys over $30 \mathrm{~mm} \mathrm{Hg}$ in $73 \%$ of the patients. Isoproterenol infusion showed $\triangle \mathrm{HR}$ over $50 / \mathrm{min}$. in $62 \%$, and a $\Delta$ BPsys fall below -20 $\mathrm{mm} \mathrm{Hg}$ in $60 \%$ of the patients. These abnormalities were noted even in the early stages of the disease.

\section{Discussion}

The analysis of the detailed questionnaire and autonomic function tests in this series of 121 patients with clinically diagnosed MSA, is the first of its kind concerning both urinary and cardiovascular systems. Urinary dysfunction has attracted less attention than postural hypotension in patients with MSA. The results of the present study, however, showed that urinary symptoms $(96 \%)$ were more common than orthostatic symptoms $(43 \%)(p<0.01)$. The most frequent urinary symptom was difficulty of voiding, in $79 \%$ of our patients, followed by nocturnal urinary frequency in $74 \%$, and the other symptoms were sensation of urgency in $63 \%$, urge incontinence in $63 \%$, diurnal urinary frequency in $45 \%$, enuresis in $19 \%$, and urinary retention in $8 \%$. The most frequent orthostatic symptom was postural faintness in $43 \%$, followed by blurred vision in $38 \%$ and syncope in $19 \%$. Previously Wenning et $a l^{16}$ mentioned urinary incontinence in $71 \%$ and urinary retention in $27 \%$, and postural faintness in $53 \%$ and syncope in $15 \%$ of their 100 clinically diagnosed cases. In our 53 patients with both urinary and orthostatic symptoms, patients who had urinary symptoms first $(48 \%)$ were more common than those who had orthostatic symptoms first (29\%), although this difference was not significant, and there were patients who developed both symptoms simultaneously (23\%). These results indicate that urinary dysfunction is more common and often an earlier manifestation than orthostatic hypotension in MSA. We also divided MSA patients into three variants, showing that the difference between urinary and orthostatic symptoms is prominent in patients with OPCA type (urinary symptoms in $92 \%$ and orthostatic symptoms in $10 \%$; $\mathrm{p}<0.01)$ and with SND type $(94 \%$ and $6 \%$, respectively; $\mathrm{p}<0.01)$ compared with those with Shy-Drager type $(100 \%$ and $82 \%$, respectively).

The results of urodynamic studies showed postmicturition residuals in $74 \%$ of our patients, and residuals over $100 \mathrm{ml}$ were noted in $52 \%$. EMG cystometry showed detrusor hyperreflexia in $56 \%$, low compliance bladder in $31 \%$, atonic curve in $5 \%$, detrusor-sphincter dyssynergia in $45 \%$, and neurogenic sphincter EMG in $74 \%$ of the patients. Bethanechol injection showed $\Delta$ Pdet over $15 \mathrm{~mm} \mathrm{H}_{2} \mathrm{O}$ suggestive of denervation supersensitivity of the bladder, ${ }^{15}$ in $19 \%$ of the patients. These findings indicate the presence of central and peripheral types of urinary dysfunction in MSA. The results seem to be relevant to the pathological lesions reported in MSA, ${ }^{17}$ which include the locus ceruleus (pontine micturition centre), ${ }^{18}{ }^{19}$ the putamen, the substantia nigra, ${ }^{20}$ sacral intermediolateral nucleus, ${ }^{21}{ }^{22}$ and Onuf nucleus, ${ }^{123}$ and, less contributary, the frontal cortex ${ }^{24-26}$ and postganglionic cholinergic fibres. ${ }^{17}$ Cardiovascular function tests showed postural hypotension below $-30 \mathrm{~mm} \mathrm{Hg}$ in $41 \%$, low CV R-R below 1.5 in $57 \%$, and supine plasma NA below $100 \mathrm{pg} / \mathrm{ml}$ in $28 \%$. Infusion of NA showed $\Delta$ BPsys over $30 \mathrm{~mm}$ $\mathrm{Hg}$ suggesting $\alpha$ supersensitivity of the vessels in $73 \%$ of the patients. Isoproterenol infusion gave a $\Delta \mathrm{HR}$ over $50 / \mathrm{min}$. suggesting $\beta 1$ supersensitivity of the heart in $62 \%$, and a $\Delta$ BPsys fall below $-20 \mathrm{~mm} \mathrm{Hg}$ suggesting $\beta 2$ supersensitivity of the vessels in $60 \%$ of the patients. These findings indicate the presence of postganglionic type cardiovascular dysfunction, and a central type of dysfunction is reported to occur in MSA. ${ }^{56}$ These results seem to be relevant to the pathological lesions reported in MSA, ${ }^{17} 2728$ which include the medullary cardiovascular centre (the solitary tract nucleus, the area reticularis superficialis ventrolateralis, the arcuate nucleus, and the dorsal vagal motor nuclei), ${ }^{29}{ }^{30}$ intermediolateral cell columns of the thoracic cord, sympathetic ganglia $^{31}$ and postganglionic adrenergic fibres. ${ }^{32}$

In conclusion, we found that urinary dysfunction is more common and often an earlier manifestation than orthostatic hypotension in MSA, although subclinical cardiovascular abnormalities appear in the early stage of the disease. The responsible sites seem to be central and peripheral for both dysfunctions.

This study was supported in part by the grants of the Japan Foundation of Ageing and Health, Ministry of Education, and Research Committee of Ataxic Diseases in the Ministry of in Neurology Ward East 11 of the Chiba University Hospital.

1 Beck RO, Betts CD, Fowler CJ. Genitourinary dysfunction in multiple system atrophy; clinical features and treatment in multiple system atrophy; clinical featu

2 Chandiramani VA, Palace J, Fowler CJ. How to recognize patients with parkinsonism who should not have urological surgery. Br F Urol 1997;80:100-4. 
3 Sakakibara R, Hattori T, Tojo M, et al. Micturitional disturbance in multiple system atrophy. Fapanese fournal of Psychiatry and Neurology 1993;47:591-98. (In English.)

4 Shy GM, Drager GA. A neurological syndrome associated with orthostatic hypotension; a clinical-pathologic study Arch Neurol 1960;2:511-27.

5 Bannister R, Mathias CJ. Clinical features and investigation of the primary autonomic failure syndromes. In: Banniste $\mathrm{R}$, Mathias CJ, ed. Autonomic failure, 3rd ed. Oxford: Oxford Medical Publications, 1992:531-47.

6 Low PA, Bannister R. Multiple system atrophy and pure autonomic failure. In:Low PA, ed. Clinical autonomic disorders. 2nd ed. Philadelphia: Lippincott-Raven, 1997:55575.

7 Dejerine J, Thomas A. L'atrophie olivo-ponto-cérébelleuse. Nouvelle iconographie de la Salpêtriére. 1900;13:30-70.

8 Adams RD, van Bogaert L, Eecken HV. Striato-nigral degeneration. I Neuropathol Exp Neurol 1964;23:584-608.

9 Quinn NP, Marsden CD. The motor disorder of multiple system atrophy. $\mathcal{F}$ Neurol Neurosurg Psychiatry 1993;56: system at.

10 The Consensus Committee of the American Autonomic Society and the American Academy of Neurology. Consensus statement on the definition of orthostatic hypotension,
pure autonomic failure, and multiple system atrophy. Neupure autonomic failure,
rology 1996;46:1470.

11 Kita K, Hirayama K. A comparative study of the autonomic disturbance in multiple system atrophy; system. Clinical Neurology (Tokyo) 1986;26:228-36. (In Japanese with English abstract.)

12 Low PA. The effect of ageing on the autonomic nervous system. In: Low PA, ed. Clinical autonomic disorders, 2nd ed. Philadelphia: Lippincott-Raven, 1997:161-75.

13 Vita G, Princi P, Calabolo R, et al. Cardiovascular reflex tests; assessment of age-adjusted normal range. $\mathcal{F ~ N e u r o l ~ S c i}$ 1986;75:263-74.

14 Abrams P, Blaivas JG, Stanton SL, et al. The standardization of terminology of lower urinary tract function; produced by the International Continence Society Committee on the International Continence Society Committee on 45 .

15 Lapides J, Friend CR, Ajemian EP, et al. Denervation supersensibility as a test for neurogenic bladder. Surgery, Gynaecology and Obstetrics 1962;114:241-4.

16 Wenning GK, Ben Shlomo Y, Daniel SE, et al. Clinical features and natural history of multiple system atrophy; an analysis of 100 cases. Brain 1994;117:835-45.

17 Daniel SE. The neuropathology and neurochemistry of multiple system atrophy. In: Bannister R, Mathias CJ, eds. Autonomic failure. 3rd ed. Oxford: Oxford Medical, 1992:564-85.
18 Blok BFM, Holstege G. A PET study on brain control of micturition in humans. Brain 1997;120:111-21.

19 Sakakibara R, Hattori T, Fukutake T, et al. Micturitional disturbance in herpetic brainstem encephalitis; contribution of the pontine micturition center (PMC). F Neurol Neurosurg Psychiatry 1998;64:269-72.

20 Yoshimura N, Sasa M, Yoshida O, et al. Dopamine D-1 receptor-mediated inhibition of micturition reflex by central dopamine from the substantia nigra. Neurourol Urodyn 1992;11:535-45.

21 Morgan C, Nadelhaft I, de Groat WC. Location of bladder preganglionic neurones within the sacral parasympathetic necleus of the cat. Neurosci Lett 1979;14:189-94

22 Skehan AM, Downie JW, Awad SA. The pathophysiology of contractile activity in the chronic decentralized feline bladder. F Urol 1993;149:1156-64.

23 Sakakibara R, Hattori T, Kita K, et al. Stress-induced urinary incontinence in patients with spinocerebellar degeneration. F Neurol Neurosurg Psychiatry 1998;64:38991.

24 Fujita T, Doi M, Ogata T, et al. Cerebral cortical pathology of sporadic olivopontocerebellar atrophy. $\mathcal{F}$ Neurol Sci 1993;116:41-6.

25 Andrew J, Nathan PW. Lesions of the anterior frontal lobes and disturbances of micturition and defaecation. Brain 1964;87:233-62.

26 Sakakibara R, Hattori T, Tojo M, et al. Micturitional disturbance after acute hemispheric stroke; analysis of the lesion site by CT and MRI. F Neurol Sci 1996;137:47-56.

27 Bennarroch EE, Smithson IL, Low PA, et al. Depletion of catecholaminergc neurons of the rostral ventral medulla in catecholaminergc neurons of the rostral ventral medulla in rol 1998;43:156-63.

28 Noda K, Katayama S, Watanabe C, et al. Decrease of neurons in the medullary arcuate nucleus of multiple system atrophy; quantitative comparison with Parkinson's disease and amyotrophic lateral sclerosis . F Neurol Sci 1997;151:89-91.

29 Yamashita A, Hirayama M, Koike Y, et al. Orthostatic hypotension caused by a localized dorsal medullary tumor. $\mathcal{f}$ Neurol Neurosurg Psychiatry 1996;61:118-19.

30 Sakakibara R, Mori M, Fukutake T, et al. Orthostatic hypotension in a case with multiple sclerosis. Clin Auton Res 1997; 7:163-5.

31 Low PA, Fealey RD. Pathological studies of the sympathetic neuron. In:Bannister R, Mathias CJ, eds. Autonomic failure. 3rd ed. Oxford: Oxford Medical, 1992:586-92.

32 Bannister R, Crowe R, Eames R, et al. Adrenergic innervation in autonomic failure. Neurology 1981;31:1501-6. 